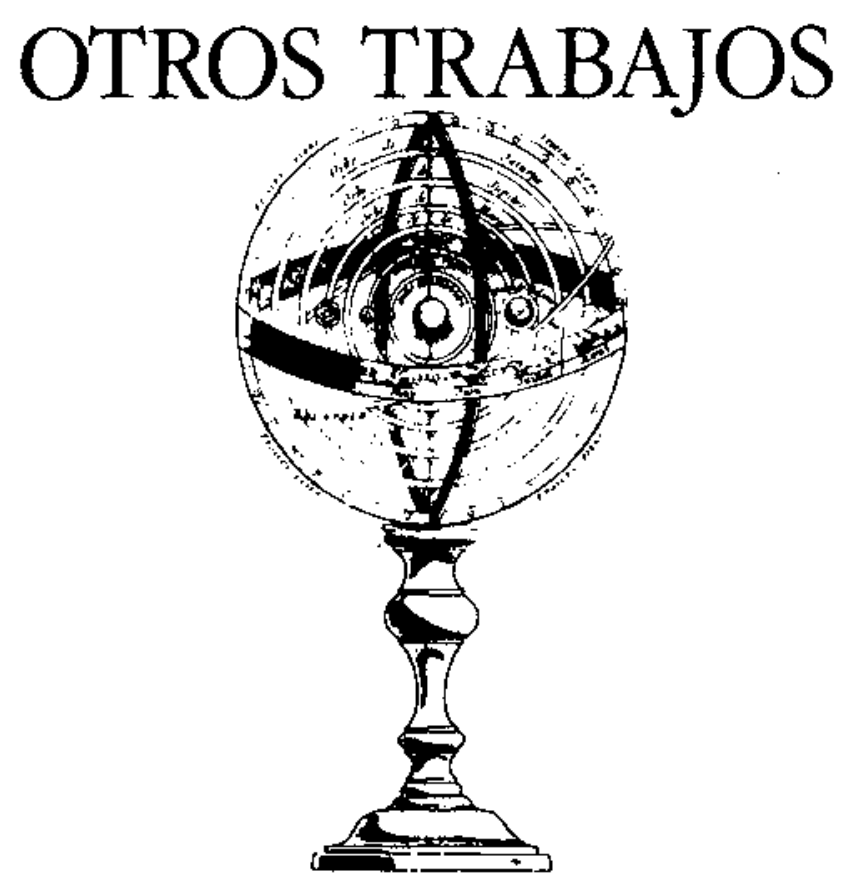

\title{
LA HISTORIA DE LA CIENCIA \\ COMO HERRAMIENTA \\ PARA LA ENSENANZA \\ DE FÍSICA EN SECUNDARIA: UN EJEMPLO EN CALOR Y TEMPERATURA
}

PESSOA DE CARVALHO, A.M. ${ }^{1}$ y CASTRO, R.S. ${ }^{2}$

'Faculdade de Educaçao. USP.

${ }^{2}$ Escueta Secundaria.

Proyecto financiado por FAPESP.

\section{SUMMARY}

In this work we suggest and analyze some stages of a course on heat and temperature which knowledge of the past of the ideas and the solution provided can enable a better understanding of the content. The hypothesis that guided the planning of activities was that the scientific content studied throughout its history comes closer to the cognitive universe, not only of the student, but of man himself

\section{INTRODUCCIÓN}

El enfoque histórico encontró, en los áltimos años, muchos adeptos que presentan los más diferentes argu- mentos para justificar su utilización en un curso de ciencias. En este trabajo, sugerimos algunos momentos 
en un curso de secundaria sobre Calor y Temperatura en los que el conocimiento del pasado de las ideas y su evolución a lo largo del tiempo podría, según nuestra expectativa y experiencia como profesores, posibilitar una mejor comprensión del contenido en cuestión.

La hipótesis que nos orientó al idear las actividades para esos momentos fue que el conterido científico estudiado a través de su historia se acerca más al universo cognoscitivo, no solamente del alumno sino del propio hombre que, antes de conocer científicamente, construye históricamente lo que conoce.

\section{NUESTRO TRABAJO}

\subsection{Objetivos}

Nuestro principal objetivo en este trabajo es sugerir momentos y situaciones en los cuales el uso de la historia parece ser adecuado y propone actividades, con un enfoque histórico, para el aula a fin de que podamos analizar, futuramente, la pertinencia, la relevancia y los efectos de esas actividades en todo el curso. Pretendemos, así, iniciar de forma concreta la aclaración del papel que este enfoque desempeña en la enseñanza de la Física.

Sabemos que en el aula las acciones y relaciones son múltiples y complejas y, por eso, el profesor tiene varias formas de tratar el tema, de facilitar una verdadera aproximación al objeto de conocimiento, utilizando los más diferentes recursos y técnicas. Uno de sus más importantes desafíos es descubrir la dosis adecuada de esos diversos acercamientos, es decir, en qué medida cada uno de los instrumentos de los cuales dispone puede contribuir a una enseñanza mejor. Para eso se hace necesario explicitar, conocer los motivos, los objetivos y las posibilidades de cada uno de ellos y, sobre todo, es necesario ensayar intentos de uso, sugerir actividades y, principalmente, analizarias en ejecución. Nuestro objetivo es, pues, iniciar ese proceso en relación con el enfoque histórico.

\subsection{Elaboración del trabajo}

Una vez elegido el contenido que trabajariamos', hicimos una relación de las principales ideas históricas sobre los conceptos incluidos en ese contenido, además de realizar varias investigaciones bibliográficas en concepciones alternativas, enfoques en textos didácticos y concepciones científicas relacionadas con el tema elegido: Calor y Temperatura.

Utilizamos inicialmente textos clásicos de la Historia de la Ciencia como, por ejemplo, Bernal (1976), Taton (1959), Hoppe (1928) y Holton (1976). Cuando profundizamos en el contexto de las ideas, buscamos obras más específicas como los trabajos del grupo de Harvard (Roller 1950), artículos que trataban de conceptos específicos y la historia de esos conceptos (Brown 1950 y 1952, Boyer 1942) y trabajos de disertación que también se preocupaban con ese enfoque, reconstruyendo cuidadosamente la historia de la termodinámica en un cierto período (Souza Filho 1987, Higa 1987). Una obra fue particularmente importante por tratarse de una recopilación. de textos originales (Magie 1935).

A partir deesa reconstrucción histórica, hecha paralelamente a la discusión del programa y del propio curso que se llevaría a cabo, profesores e investigadores de esa investigación, sugerimos algunos momentos y formas de uso para el material que elaboramos.

\subsection{Actividades}

Se podría haber tratado la historia de los conceptos en el aula de varias maneras, entre ellas, a través de clases expositivas, sesiones de vídeo, trabajos de investigación bibliográfica o lecturas. Optamos, la mayor parte de las veces, por utilizar el canal de la lectura por considerar oportuno recuperarla como actividad importante en la búsqueda del conocimiento. Una actividad, sin embar* go, se elaboró bajo la forma de orientación de clase: un «guión de discusión» elaborado a partir de un texto original que juzgamos muy largo para que los alumnos lo leyeran.

Presentaremos a continuación tres de las actividades que elaboramos con un breve comentario sobre cada una de ellas.

\section{Primer ejemplo}

Esta actividad surgió a partir del análisis de una clase, grabada en vídeo, en la cual los alumnos discutían -entre otros hechos relacionados con el estudio del calor y la temperatura- la existencia de un nivel fijo de temperatura durante el proceso de ebullición. Una alumna, reacia en aceptar tal hecho, sugirió que se averiguara experi* mentalmente, lo que nos remitió a un fragmento de un texto de Daniel Gabriel Fahrenheit, en el cual relata su sorpresa y deseo de constatar ese hecho cuando tomó conocimiento de él.

El texto distribuido a los alumnos contenía una pequeña introducción que situaba las palabras del propio científico en su trabajo (Magie 1935, pp. 131-132).

"Daniel Gabriel Fahrenheit (1686-1736), célebre fabricante de instrumentos meteorológicos, hizo una serie de experimentos con sus termómetros, entre los cuales había uno que averiguaba la existencia del nivel de temperatura en el punto de ebullición del agua, según podemos constatar en este texto escrito por él mismo:

"Hace casi diez años leí, en una edición de Historia de la Ciencia de la Real Academia de París, que el célebre Amontons, usando un termómetro que él mismo había inventado, había descubierto que el agua hierve a un grado fijo de calor. Yo ya estaba estimulado, con un deseo enorme de hacerme un termómetro del mismo tipo, de forma que yo pudiese, con mis propios ojos, 
observar ese bello fenómeno de la naturaleza y convencerme de la verdad del experimento.

"Así es que intenté construir un termómetro pero, đebido a mi falta de experiencia en la construcción, mis esfuerzos fueron vanos [...]. Yo aplacé otras repeticiones de mis intentos para un momento más conveniente. Aunque me faltasen posibilidades y tiempo, mi entusiasmo no disminuyó y yo seguía deseoso de ver el resultado del experimento. Fue cuando me vino a la mente lo que ese cuidadoso observador de los fenómenos naturales había escrito sobre la corrección del barómetro: él había observado que la altura de la columna de mercurio en el barómetro se alteraba un poco en virtud de la variación de la temperatura del mercurio. A partir de eso, deduje que, quizá, se pudiera construix un termómetro con mercurio, lo que no sería difícil de hacer. Usándolo, podría ser factible llevar a cabo el experimento que yo tanto deseaba realizar.

"Cuando se hizo un termómetro de ese tipo (quizá imperfecto de varias maneras), el resultado respondió a mis expectativas $y$, con gran placer intelectual, observé la verdad del hecho.

„Fahrenheit comprobó, además, otros líquiđos y observó que ellos también poseían puntos de ebullición fijos, bajo condiciones normales o, según sus propias palabras, "hervían a otros grados fijos de calor"."

Esa actividad, a pesar de que el texto no contiene muchas informaciones que puedan colaborar al perfeccionamiento de conceptos y explicaciones, puede servir como pretexto para una discusión sobre la estructura de la actividad científica: el cambio de informaciones entre investigadores, la necesidad de comprobar datos, eI avance de la técnica en función de las cuestiones de la ciencia, etc. Reflexionar sobre la ciencia a través del diálogo establecido entre la expectativa del alumno y la declaración del científico nos parece oportuno y fructífero en la medida en que aproxima las lecturas que ellos hacen del mundo.

\section{Segundo ejemplo}

Esta actividad se hizo a partir de un fragmento de una conferencia realizada por el médico Joseph Black, también químico, que hacia 1760 sugirió la necesidad de distinguircalor de temperatura. En esa conferencia él discute varias situaciones de mezclas y construye la distinción de esos dos conceptos, a pesar de confundirlos, todavía, algunas veces.

El guión se presenta bajo la forma de preguntas generadoras de ideas o problemas abiertos que expone el profesor. A continuación, el grupo de alumnos los discute hasta que se llegue a la solución. La orientación histórica, unida a la dinámica de discusión en equipo, nos pareció una buena manera de secuenciar el proceso continuo de persecución y construcción del objeto del conocimiento.
- ¿Calor y temperatura representan lo mismo?

- Llamar la atención hacia los objetos que hay alrededor y preguntar sobre el equilibrio térmico.

- Si se utiliza un buen termómetro para averiguar la temperatura de esos cuerpos, ¿qué se constatará?

- ¿Podemos decir que tienen la misma temperatura? ¿Y que tienen el «mismo calor» o «calores iguales»?

- Si cada uno de nosotros elige un objeto y lo sujeta entre las manos durante algún tiempo, ¿qué ocurrirá? ¿Podemos garantizar que después de algún tiempo todos los objetos estarán a la temperatura de nuestro cuerpo? ¿Esos cuerpos gastarán el mismo tiempo para alcanzar esa temperatura o cada uno tendrá un tiempo propio?

- Proponer el problema abierto:

Cuando calentamos dos líquidos diferentes, ambos a la misma temperatura inícial de $20^{\circ} \mathrm{C}$, ¿cuál de ellos llega primero a la temperatura de $50^{\circ} \mathrm{C}$ ?

¿Qué hay que saber sobre esos líquidos para contestar a esa pregunta?

Y si los líquidos fuesen los mismos, ¿qué determinaría la rapidez del calentamiento?

Observa la siguiente situación:

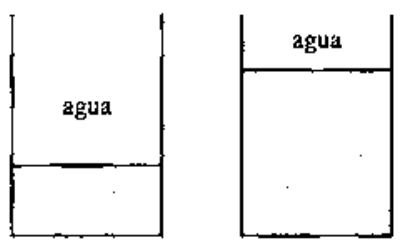

El segundo recipiente tiene el doble de agua que elprimero. Inicialmente ambos están a temperatura ambiente. Se calientan, con mecheros idénticos, hasta que alcancen Ia temperatura de $50^{\circ} \mathrm{C}$.

¿Podemos afirmar que el agua del primer recipiente tardará menos para alcanzar esa temperatura? ¿Qué significa esa diferencia de tiempo necesaria para que sufra una misma variación de temperatura?

¿Y si cambiamos el problema y fijamos el tiempo de exposición a la llama? Es decir, si consideramos los mecheros idénticos y se mantienen los vasos sobre ellas durante un mismo intervalo de tiempo, icrees que la temperatura del agua de los dos recipientes será la misma?

Al fijar el tiempo de exposición a la llama y garantizando que los mecheros son iguales, ¿no suponemos la igualdad de otra cosa? ¿Qué? 
Sistematizando: En la primera situación, las cantidades de agua reciben diferentes cantidades de calor de los mecheros, pues se mantienen expuestas a ella por tiempos diferentes, pero alcanzan una misma temperatura. En la segunda situación, las cantidades de agua reciben la misma cantidad de calor alcanzando diferentes tempera turas. Así, ¿calor y temperatura son la misma cosa?

Y si los líquidos son diferentes, ¿qué podemos concluir?

Se debe dirigir la discusión a que se establezca la dependencia de la cantidad de calor con la masa, el material (calor específico) y la variación de temperatura. Por lo tanto, se debe concluir que, si no hay diferencia de temperatura no hay cambio de calor. La cuestión generadora de la discusión es la siguiente:

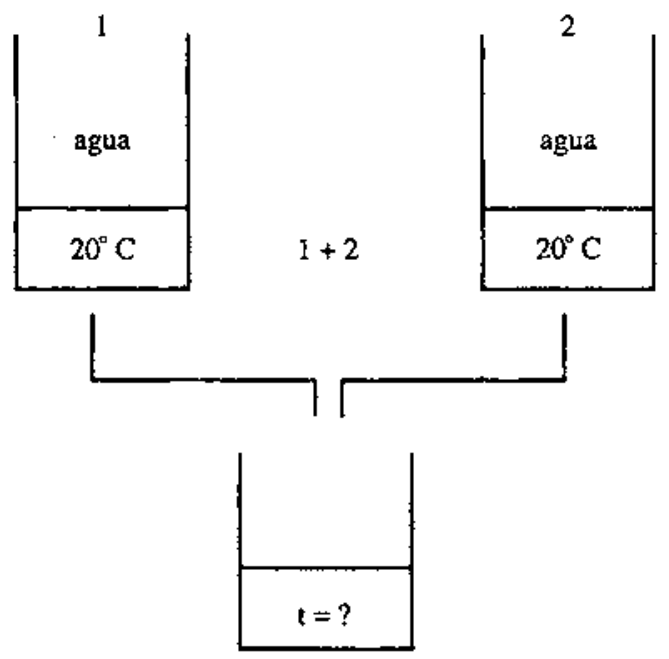

¿Qué pasará ahora, en función de la diferencia de temperatura? ¿En cuántos grados se aumenta la temperatura del agua más fría?

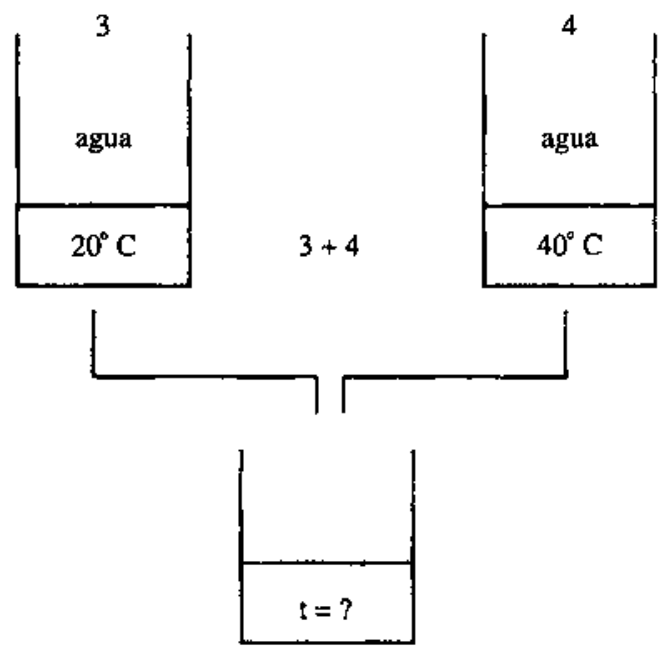

¿Y en cuántos grados disminuye la temperatura del agua más caliente? Esa variación en la temperatura de las aguas caliente y fría isería la misma si la cantidad de agua no fuese la misma en los dos recipientes? Si hacemos:
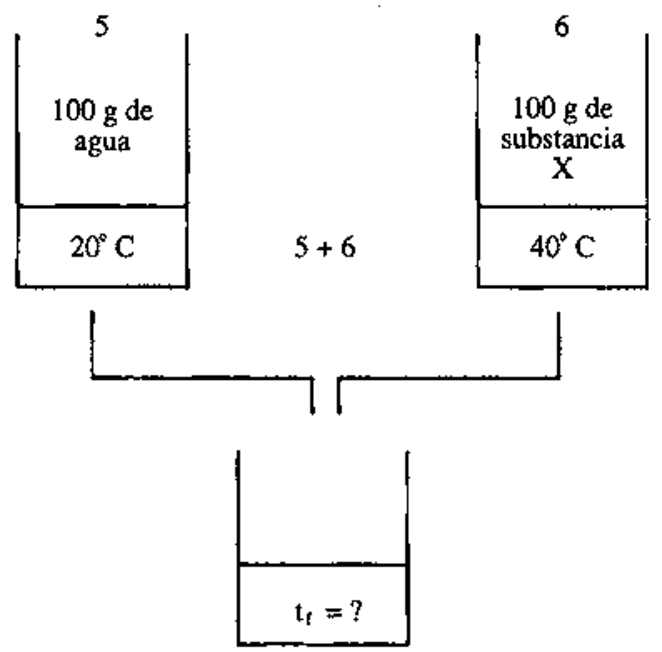

Si el calor absorbido por el agua es igual al calor cedido por la substancia $X$, ¿crees correcto esperar que ambos, el agua y la substancia $X$, sufran la misma variación de temperatura? ¿Por qué?

Si la temperatura final de la mezcla es a $28^{\circ} \mathrm{C}$, zcuál es la variación de temperatura que sufre el agua? i $\mathrm{Y}$ la substancia X? Si las masas son iguales y la cantidad de calor que el agua recibe es igual a la cantidad de calor que la substancia $X$ cede, algún factor unido a la naturaleza de la substancia (agua y $X$ ) compensará esa diferencia. A ese factor, unido al material, que es una característica suya, lo liamamos calor específico.

Claro está que el profesor puede modificar el guión presentado a lo Iargo de la clase. Lo importante, sin embargo, es que no se dejen a un nivel secundario cuestiones aparentemente triviales, raciocinios que nos parecen obvios pero que la historia nos muestra, a través de los textos de los propios científicos, que han tenido influencia considerable en la evolución de la ciencia y que han sido fuente de producción de conocimiento.

\section{Tercer ejemplo}

Después de esa clase de discusion de ideas y de cuestiones-clave, se realizarian experimentalmente situaciones análogas a éstas y, en seguida, los alumnos recibirían el siguiente texto, también extraído de la conferencia de Joseph Black:

«El segundo avance en nuestro conocimiento sobre el calor, que se ha alcanzado por el uso de termómetros, es la noción que tenemos, ahora más que antes, de la distribución de calor entre diferentes cuerpos. 
"Observé anteriormente que, aunque sin la ayuda de termómetros, podemos notar la tendencia del calor en difundirse de algún cuerpo caliente hacia un cuerpo más frío, próximo a él, hasta distribuirse entre ellos de tal forma que ninguno puede sacar más calor del otro. [...] Averiguamos que cuando toda acción mutua termina, un termómetro puesto en cualquiera de los cuerpos adquiere el mismo grado de expansión: por eso su temperatura es la misma y el equilibrio es universal.

"Por el uso de esos instrumentos aprendimos que, si tomamos mil o más tipos diferentes de materiales, tales como metales, piedra, sal, madera, corcho, pluma, lana, agua y una variedad de otros fluidos, aunque están todos, a principio, a diferentes calores, y si los dejamos juntos en una misma sala sin calefacción y sin iluminación del sol, el calor se comunicará de los cuerpos más calientes a los más fríos durante algunas horas o en el transcurso de un día. Al final de ese tiempo, si usamos un termómetro, veremos que ellos están exactamente en el mismo grado. El calor, por lo tanto, se distribuye en esa ocasión hasta que ninguno de esos cuerpos tenga una mayor demanda o atracción al calor que cualquiera de ellos tenga; en consecuencia, cuando usamos un termómetro sucesivamente, después que el primero ha reducido la temperatura del instrumento a la suya propia, ninguno de los otros aumentará o disminuirá la cantidad de calor que el primero dejó en él. Eso es lo que se ha llamado normalmente "calor igual" o "igualdad de calor" entre cuerpoś diferentes: yo llamo a eso equilibrio de calor.

"La naturaleza de ese equilibrio no fue comprendida hasta que yo indiqué un método para investigarla. EI Dr. Boerhaave supuso que, cuando se obtiene, hay una cantidad de calor igual en cualquier medida igual de espacio, aunque éste se rellene por diferentes cuerpos. El profesor Musschenbroeck expresa su opinión de la misma forma. [...] La razón para que ellos tengan esa opinión es que, para cualquiera de esos cuerpos el termómetro usado indica el mismo grado.

"Pero eso es aceptar una visión precipitada sobre el tema. Cantidades de calor se confunden en diferentes cuerpos con su fuerza o intensidad, a pesar de ser evidente que existen dos cosas distintas que deberían distinguirse cuando pensamos en la distribución del calor." (Magie 1935, pp.134-135).

\section{REFERENCIAS BIBLIOGRÁFICAS}

BERNAL, J.D., 1976. Ciência na História. Lisboa, Coleçäo Movimento, Livros Horizonte, Vol. 1 y 3.

BOYER, C.B., 1942. Early Principles in the Calibration of Thermometers, Am. Journal of Physics, 10(4), pp. 176-180.
De nuevo, la aproximación entre la manera de interrogar del alumno y del científico al objeto en estudio puede ser fructífera y auxiliar al alumno en la elaboración y en el refinamiento de los conceptos. Al tener acceso a los estadios del desarrollo de las ideas científicas y no sólo estar limitado al producto final de ellas, los alumnos pasan a ver la ciencia como actividad dinámica, y la construcción del conocimiento como algo posible, incluso para ellos puesto que, ahora, la despliegan en un proceso. $Y$ como conocer es acercarse cada vez más al objeto, aunque cada acercamiento puede ocurrir siguien. do rumbos o «historias» diferentes, lo que se generará como efecto es el objeto en construcción, sea por el alumno, sea por los científicos a lo largo de la historia. Conocer las etapas del desarrollo de una idea o de un concepto desmitifica la ciencia en cuanto asunto prohibido a los no iniciados, y acerca el discurso científico al discurso que el alumno comprende. Así, pensar la ciencia pasa a ser posible y se descubre que producir conocimiento empieza con un sencillo acto común y posible para todos: pensar.

Tal vez sea ésa la más importante contribución que el enfoque histórico puede propiciarnos, a nosotros, profe. sores, y a nuestros alumnos: el saber científico, aunque sofisticado, es producto de la actividad humana. Así, las limitaciones de la ciencia se reconocen al mismo tiempo en que se revela no sólo la posibilidad de participación de todos en la edificación del conocimiento, sino, principalmente, la necesidad de que todos se conviertan en agentes activos de la construcción y control de lo que buscan conocer.

\section{NOTA}

${ }^{3}$ Calor y Temperatura fue tema de algunos trabajos de nuestro grupo en la «Faculdade de Educaçao de USP» que acept 6 Ia sugerenciaproferida durante el Primer Encuentro Latinoamericano de Grupos de Investigación en Enseñanza de Física (México, 1988) de explotar este contenido de forma conjunta.
BROWN, S.C., 1950. The caloric theory of heat, Am. Journal of Physics, 18, pp. 367-373.

BROWN, S.C., 1952. Count Rumford's Concept of Heat, Am. Journal of Physics, 20, pp, 331 334 . 
HIGA, T.T., 1987. Conservaçäo da Energia: Estudo Histórico e Levantamento Conceitual dos Alunos. Dissertao de Mestrado orientada por A. I. Hamburger, IFUSP/FEUSP. São Paulo.

HOLTON, G., 1976. Introducción a los conceptos y teorias de las ciencias fisicas, revisada y ampliada por S.G. Brush, (Editorial Reverté: Barcelona).

HOPPE, E., 1928. Histoire de la Physique. (Payot: París).

MAGIE, W.F., 1935. A Source Book in Physics, (McGraw-Hill Book Company: Nueva York y Londres\}.
ROLLER, D., 1950. The early development of the concepts of temperature and heat - decline of the caloric theory. (Harvard University Press: Cambridge).

SOUZA FILHO, O.M., 1987. Evoluçao da idéia de conservaçao da energia: um exemplo da historia da ciência no ensino de física. Dissertao de mestrado orientada por M. R. Robilota, IFUSP/FEUSP. São Paulo.

TATON, R., 1959. História Geral das Ciências. (Difuso Européia do Livro: Săo Paulo). 